\title{
Bone turnover in early rheumatoid arthritis. 2. Longitudinal bone density studies
}

\author{
P N SAMBROOK, * B M ANSELL, S FOSTER, J M GUMPEL, R HESP, \\ AND J REEVE
}

From the MRC Clinical Research Centre and Northwick Park Hospital, Watford Road, Harrow, Middlesex

SUMMARY Serial measurements of bone mineral in 17 ambulant female patients with rheumatoid arthritis (RA) of recent onset and 19 age matched female controls were made in the radius by computed tomography and in the vertebrae by dual photon absorptiometry. Loss of trabecular bone from the distal radius was more rapid in RA $(p=0.0014)$, but there was no difference in the $i$ rate of loss of bone mineral from the radial midshaft or lumbar spine compared with the controls.

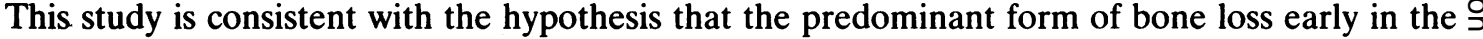
disease is in the vicinity of affected joints.

Key words: osteoporosis, bone densitometry.

Osteoporosis of the subchondral bone of involved joints is well recognised in rheumatoid arthritis (RA) and forms part of the diagnostic criteria of the disease. ${ }^{1}$ In addition, a more generalised involvement of the axial and appendicular skeleton may occur. $^{2-4}$ Juxta-articular bone loss occurs early in the disease, being evident in some patients only a few months after the onset of symptoms, ${ }^{5}$ but whether bone is lost early in the axial skeleton and in the appendicular skeleton at sites away from involved joints is unknown.

In the last decade more accurate quantitative methods of assessing bone mass in vivo have become available. $^{6}$ Some of these techniques have been applied in RA but generally in cross-sectional studies at appendicular sites and usually in patients with longstanding disease. ${ }^{3}$ 7-10

In the present study we have performed serial measurements of bone density in the distal radius, radius midshaft, and lumbar spine of ambulant outpatients with RA of recent onset and a group of age and sex matched controls. Since among RA patients who have not been treated with corticosteroids postmenopausal women are the group most at risk of developing osteoporosis, ${ }^{2}{ }^{11}$ we selected for

\footnotetext{
Accepted for publication 2 April 1985.

Correspondence to Dr J Reeve, Bone Disease Research Group, Clinical Research Centre, Watford Road, Harrow, Middlesex HA1 3UJ.

*Present address: Garvan Institute, St Vincent's Hospital, Sydney, NSW 2010, Australia.
}

study predominantly postmenopausal women wi had recently developed RA. In the companion paper we have measured biochemical and kinetre markers of bone metabolism in these subjects. ${ }^{12}$

\section{Patients and methods}

\section{S U B JE C TS}

Seventeen female patients who had recently developed classical or definite seropositive $R^{1}$ and a group of 19 age matched female controls were studied. Details of the subjects and the methods used to assess their disease activity are described in 0 the companion paper. ${ }^{12}$ The wrist joints were clinically involved in all the patients. The indexes of $\delta$ disease activity measured were the Ritchie articular index,${ }^{13}$ the joint count (number of active joints), 을 grip strength (mean of three recordings, dominant hand), erythrocyte sedimentation rate, and the C-reactive protein concentration. Physical activity was measured subjectively by the Framingham $\%$ activity index. ${ }^{14}$

\section{DENSITOMETRIC STUDIES}

Bone density was measured in the dominant radius

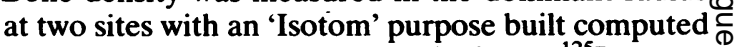
tomography densitometer employing a ${ }^{125}$ I source $(29 \mathrm{keV}, 7 \cdot 4-18.5 \mathrm{GBq})$ as previously described. ${ }^{15} \mathrm{O}$ In the distal radius trabecular bone density (TBD, $\stackrel{\circ}{\circ}$ $\mathrm{cm}^{-1}$ ) was measured at $8-10 \%$ of the distance 
between the ulnar styloid and olecranon as the mean linear attenuation coefficient of the central $50 \%$ of the bone cross section. To avoid cortical bone in this site only the inner $50 \%$ of the bone cross section was measured. At the junction of the distal one-third and proximal two-thirds of the radial midshaft cortical bone was measured as total absorption $(\mathrm{cm})$ calculated as the mean linear attenuation coefficient of the whole cross section multiplied by the crosssectional area. The radiation dose with this method was $0.02 \mathrm{mSv}$ at the measurement site, and the coefficient of variation for repeat measurements in normal subjects was $2 \% .{ }^{15}$ The cross-sectional area of the radius was recorded at each measurement to ensure that serial measurements were performed as close as possible to the original site.

Bone mineral content (BMC) in the lumbar spine was measured by a Novo 22a dual photon absorptiometer with a ${ }^{153} \mathrm{Gd}$ source $(44 \mathrm{keV}, 100 \mathrm{keV}, 37$ $\mathrm{GBq})$ as previously described. ${ }^{16}$ Scans were made of the second to fourth lumbar vertebrae, and bone mineral was expressed as the mean lumbar BMC $(\mathrm{g} / \mathrm{cm})$ of this region. The radiation dose with this method to the skin was $0 \cdot 1 \mathrm{mSv}$ and to the uterus about $10 \mu \mathrm{Sv}$. The coefficient of variation of repeat measurements in normal postmenopausal women was previously reported as $2 \cdot 6 \% .{ }^{16}$ In our hands, based on the standard deviations about the individual regressions of the subject included in this study, the mean coefficient of variation was $1.8 \%$.

Serial measurements were made by these methods every three to four months for a mean period of 1.24 years (range 0.9-1.6).

\section{DATA ANALYSIS}

Serial bone mineral values for the radius and lumbar spine were fitted by linear regression for each subject, and the slope and intercept on the regression line were used to determine the percentage change from baseline values over 12 months.

The statistical significance of the changes between the two groups at each site was compared by a Mann-Whitney U test. Parametric correlation analyses of longitudinal data were performed against measures of disease activity in the radius midshaft and lumbar spine. Because distal radius loss was not normal in distribution, correlations at this site were performed by Spearman's rank correlation.

\section{Results}

The trends in bone density in the distal radius, radial midshaft, and lumbar spine for the two groups are shown in Figs 1-3. In the radial midshaft and lumbar spine inspection of the data confirmed that a linear fit was satisfactory. In the distal radius, most data

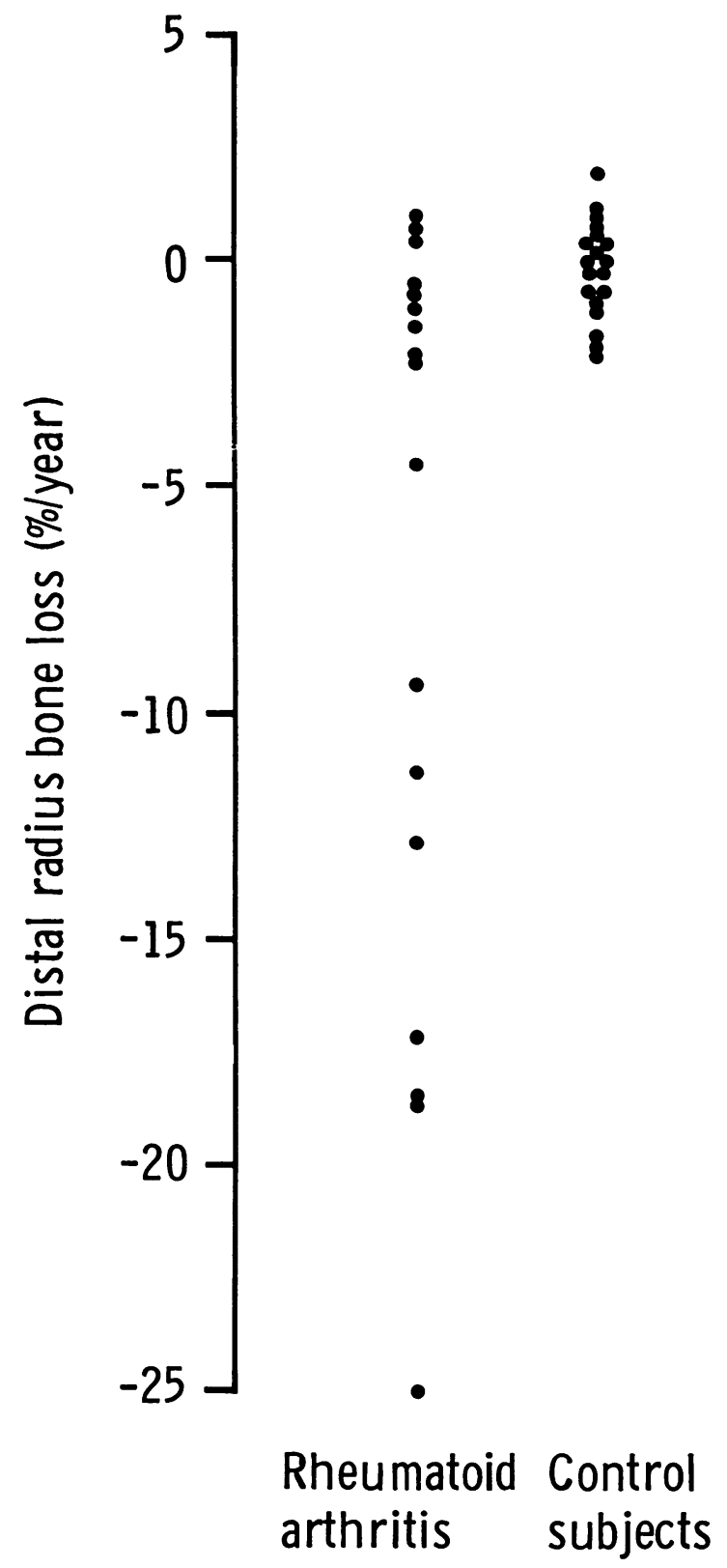

Fig. 1 Trends in trabecular bone density in the distal radius.

were again best fitted to a linear regression, but some patients showed a rapid bone loss in a stepwise fashion (Fig. 4). Calculation of the rate of loss from the distal radius in these patients as the difference between initial and final values gave similar results 


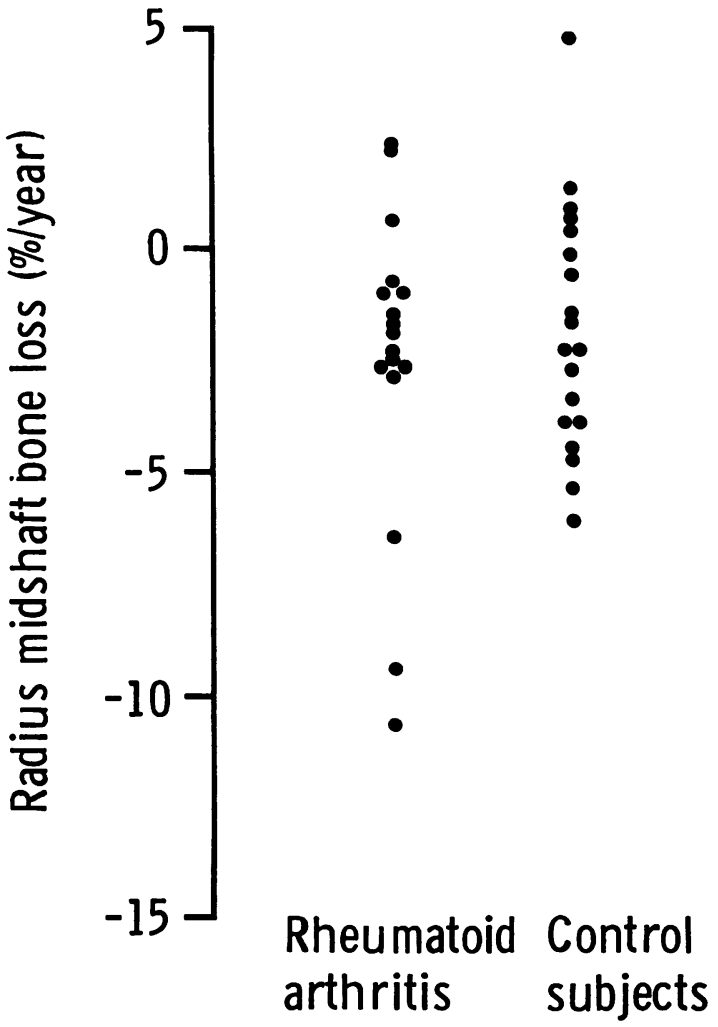

Fig. 2 Trends in cortical bone density in radial midshaft.

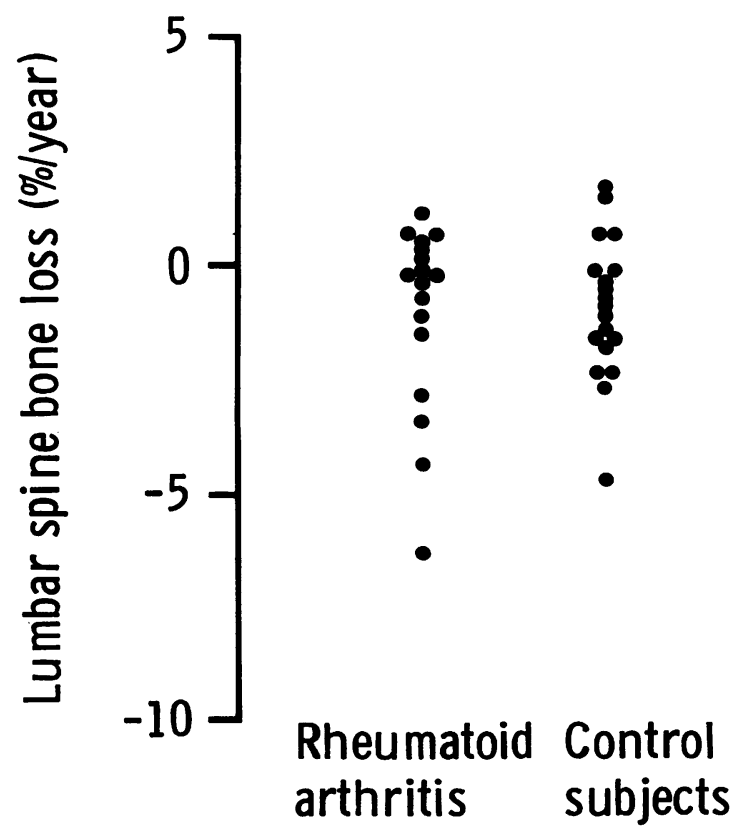

Fig. 3 Trends in bone mineral content of lumbar spine.

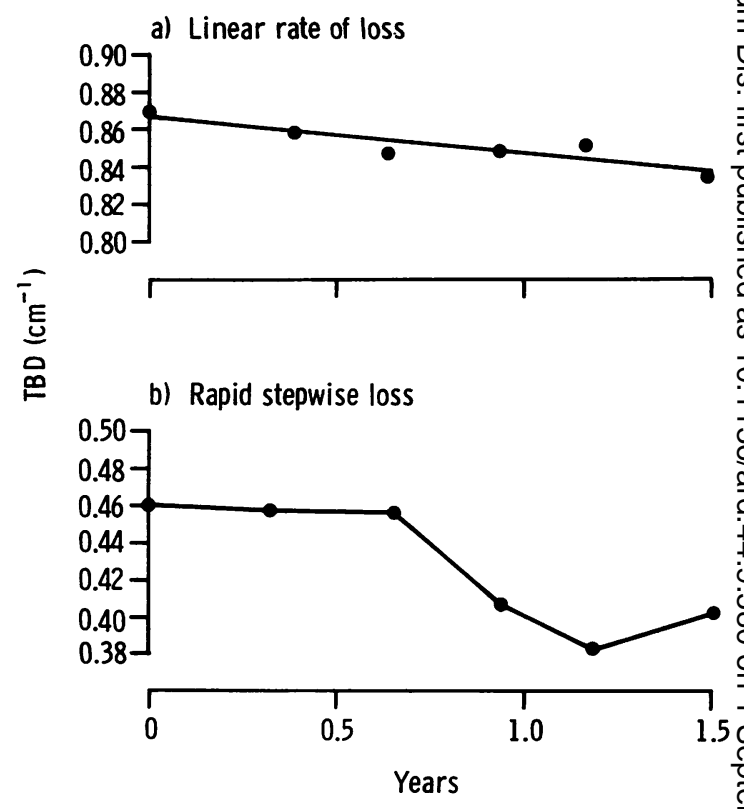

Fig. 4 Examples of trabecular bone loss from the distal radius in individual patients with rheumatoid arthritis.

to those obtained with a linear regression, so a line fit was used for all subjects.

There was no significant difference in the rate of bone loss in the radial midshaft or lumbar spine between the two groups, but in the distal radius patients with RA lost trabecular bone significantly faster than controls $(p=0.0014)$.

Two measures of disease activity correlated with the rate of lumbar spine bone loss but not with bone loss from the radius. The significant correlations were with joint count $(r=0.64, p<0.01)$ and $C$ reactive protein $(\mathrm{r}=0.52, \mathrm{p}<0.05)$.

\section{Discussion}

In this study we selected patients with RA of recent onset for two reasons. Firstly, we sought to determine whether there was accelerated bone loss early $\frac{D}{0}$ in the course of the disease from the axial and appendicular skeleton at sites not affected by $\mathscr{N}$ juxta-articular osteoporosis. Secondly, we wanted to $N$ avoid the complicating additive effects of severe N immobility and corticosteroid therapy on bone loss.

The contribution of immobility to bone loss in RA has long been recognised but is difficult to quantify. Castillo et al. ${ }^{17}$ evaluated hand radiographs in RA and found that cystic changes were more common and osteoporosis less common in men, while in women the reverse was true. The greater degree of $\underset{\mathbb{D}}{ }$ 
osteoporosis in women was attributed to diminished physical activity, while in men cystic erosions and less osteoporosis were attributed to a higher grade of manual activity. Grip strength has been found to correlate with cortical bone thickness of the phalanges in the hands of patients with $\mathrm{RA},{ }^{18}$ and Hancock et al. ${ }^{9}$ found that reductions in bone mineral in the femoral midshaft of patients with RA correlated with decreased walking time and increased functional grade. Selection of ambulant patients with disease of recent onset does not completely correct for restricted physical activity in RA $^{12}$ but reduces this effect.

The finding of accelerated loss of trabecular bone from the distal radius in RA but not from the radial midshaft or lumbar spine suggests that predominantly local factors mediate bone loss early in the disease. It can be seen from Fig. 1 that rates of loss are quite heterogeneous in the patients with RA. The difference between patients and controls at this site cannot be explained by the inappropriate use of linear regressions, since calculation of the bone loss as the difference between initial and final values gave similar results. Rapid stepwise loss of trabecular bone from the distal radius has previously been described in osteoporotic patients by Ruegsegger $e t$ al. ${ }^{19}$ Fluctuation in disease activity may contribute to a similar phenomenon in RA. The lack of correlation between this loss and measures of disease activity suggests that global measures of disease activity poorly reflect what is occurring in individual joints such as the carpus.

Two sources of error may have influenced the determination of bone loss at the distal site in our study. Firstly, trabecular bone is not homogeneously distributed. ${ }^{20} \mathrm{~A}$ high reproducibility for TBD can therefore only be attained if identical samples are examined in serial measurements. ${ }^{20}$ This was achieved in our study by multiple measurements of juxtaposed sections, recording of the cross-sectional area of bone sections to identify the correct site for repeat measurements, and exclusion of measurements with movement artefacts. A second possible source of error is that values obtained for TBD overestimate trabecular bone since they incorporate a significant contribution from marrow, and changes in the fat content of marrow will affect TBD. ${ }^{21}$ Yellow marrow (fat) has a lower linear attenuation coefficient than red marrow (haematopoietic), and a $10 \%$ change from red to yellow marrow will alter the linear attenuation coefficient to the same degree as a $0.5 \%$ change in bone density. ${ }^{20}$ However, as haematopoietic marrow disappears from the peripheral skeleton by age 25 and as changes in subchondral bone marrow fat have not been observed in RA, ${ }^{22}$ this is unlikely to explain the changes we found.
There are few previous longitudinal studies of bone mass in RA. Virtama et al. ${ }^{18}$ compared cortical indexes from hand $x$-rays at an interval of four years and found that progression of osteoporosis was more rapid early in the disease. These indexes are reasonable measures of cortical bone status but give no information about trabecular bone and may reflect localised rather than generalised skeletal changes. Oka et al. ${ }^{23}$ measured bone mineral serially in the forearm of patients with RA of recent onset and found an average decrease of $3 \%$ per year, which was not considered significant. This study employed single energy photon absorptiometry, which does not discriminate between cortical and trabecular bone. The computed tomographic technique we used allows direct measurement of trabecular bone and is considerably more sensitive.

The rate of loss of bone mineral observed in the radial midshaft and lumbar spine does not support any generalised loss of bone mineral early in the disease. The radial midshaft comprises predominantly cortical bone, which turns over more slowly than trabecular bone, so that the observation period may have been insufficient to detect a difference between the two groups at this site. The lumbar spine contains $50 \%$ trabecular bone, making it more sensitive as a measurement site. To show a significant $(\alpha=0.05)$ difference in the rate of loss of BMC in the lumbar spine with a certainty of $95 \%$ $(1-\beta=0.95)$ in the present study, given the observed measurement precision and 18 patients in each group followed every three months for 1.24 years, a $2.5 \%$ difference in the annual rate of loss between the groups would be required. ${ }^{24}$ Consequently, accelerated loss from the axial skeleton in RA of this magnitude cannot be excluded without a longer observation period, larger sample size, or increased frequency of measurement.

This work was supported by the Australian Arthritis and Rheumatism Foundation and the Arthritis and Rheumatism Council of Great Britain.

\section{References}

1 Ropes M W, Bennett C A, Cobb S, et al. Proposed diagnostic criteria for rheumatoid arthritis. Ann Rheum Dis 1959; 18: 49-53.

2 Saville P D, Kharmosh O. Osteoporosis of rheumatoid arthritis: influence of age, sex and corticosteroids. Arthritis Rheum 1967; 10: $423-30$.

3 Bjelle A O, Nilsson B E. Osteoporosis in rheumatoid arthritis. Calcif Tissue Res 1970; 5: 327-32.

4 Kennedy A C, Smith D A, Anton H C, Buchanan W W. Generalized and localized bone loss in patients with rheumatoid arthritis. Scand J Rheumatol 1975; 4: 209-15.

5 Bywaters E G L. The early radiological signs of rheumatoid arthritis. Bull Rheum Dis 1960; 11: 231-4.

6 Cohn S H. Techniques for determining the efficacy of treatment of osteoporosis. Calcif Tissue Int 1982; 34: 433-8. 
7 Mueller M N, Jurist J M. Skeletal status in rheumatoid arthritis. Arthritis Rheum 1973; 16: 66-70.

8 Christiansen C, Rodbro P. Skeletal status in patients with rheumatoid arthritis. Acta Med Scand 1975; 198: 453-4.

9 Hancock D A, Aseidu-Offei S, Atkinson P J, Reed G W, Wright V. Femoral bone loss in patients with rheumatoid arthritis and osteoarthrosis. Rheumatol Rehabil 1978; 17: 65-71.

10 Als O S, Christiansen C, Hellesen C. Prevalence of decreased bone mass in rheumatoid arthritis. Relation to antiinflammatory treatment. Clin Rheumatol 1984; 3: 201-8.

11 Reid D M, Kennedy N J S, Smith M A, Tothill P, Nuki C. Total body calcium in rheumatoid arthritis: effects of disease activity and corticosteroid treatment. Br Med J 1982; 285: 330-2.

12 Sambrook P N, Ansell B M, Foster S, et al. Bone turnover in early rheumatoid arthritis. 1. Biochemical and kinetic indexes. Ann Rheum Dis 1985; 44: 575-9.

13 Ritchie D M, Boyle J A, McInnes J M. Clinical studies with an articular index for the assessment of joint tenderness in patients with rheumatoid arthritis. $Q J$ Med 1968; 147: 393-406.

14 Kannel W B, Sorlie P. Some health benefits of physical activity. The Framingham study. Arch Intern Med 1979; 139: 857-61.

15 Elsasser U, Reeve J. Bone density measurements with computed tomography. Br Med Bull 1980; 36: 293-6.

16 Krolner B, Pors Nielsen S. Measurement of bone mineral content (BMC) of the lumbar spine. I. Theory and application of a new two dimensional dual photon attenuation method. Scand J Clin Lab Invest 1980; 40: 653-63.

17 Castillo B A, El Sallab R A, Scott J T. Physical activity, cystic erosions and osteoporosis in rheumatoid arthritis. Ann Rheum $\vec{\Rightarrow}$ Dis 1965; 24: 522-7.

18 Virtama $P$, Helella $T$, Kalliomaka $J$ L. Osteoporosis in rheumatoid arthritis: a follow up study. Acta Rheumatol Scand 1968; 14: 276-84.

19 Ruegsegger P, Dambacher M, Anliker M. Longitudinal evalua- $\frac{\bar{S}}{\partial}$ tion of normal and osteoporotic bone loss in women by means $\stackrel{\unrhd}{\varrho}$ of quantitative CT. J Comput Assist Tomogr 1983; 7: 557-8.

20 Ruegsegger P, Stebler B, Dambacher M. Quantitative com- $\omega$ puted tomography of bone. Calcif Tissue Int 1982; 34: 96-103. $\vec{\circ}$

21 Mazess R B. Errors in measuring trabecular bone by computed tomography due to marrow and bone composition. Calcif $\overrightarrow{\vec{\omega}}$ Tissue Int 1983; 35: 148-52.

22 Lereim P, Goldie I F. Relationships between morphologic features and hardness of the subchondral bone of the medial $\bar{\alpha}$ tibial condyle in the normal state and in osteoarthritis and + rheumatoid arthritis. Arch Orthop Trauma Surg 1975; 81: 1-11.

23 Oka M, Reckonen A, Kuikka J, Anttinen J. Bone mineral density in rheumatoid arthritis measured by the gamma $\infty$ transmission method. Scand J Rheumatol 1975; 4: 28-32.

24 Schlesselman J J. Planning a longitudinal study. II. Frequency of measurement and study duration. J Chronic Dis 1973; 26: 561-70. 